\title{
Rapid solidification of acoustically levitated Al-Cu-Si eutectic alloy under laser irradiation
}

\author{
YAN Na, GENG DeLu, HONG ZhenYu \& WEI BingBo* \\ Department of Applied Physics, Northwestern Polytechnical University, Xi'an 710072, China
}

Received October 15, 2010; accepted January 10, 2011

\begin{abstract}
Al-27\% Cu-5.3\% Si ternary eutectic alloy was melted using a YAG laser and then solidified while being acoustically levitated. A maximum undercooling to $195 \mathrm{~K}\left(0.24 T_{\mathrm{L}}\right)$ was achieved with a cooling rate of $76 \mathrm{~K} / \mathrm{s}$. The solidification microstructure was composed of $(\mathrm{Al}+\theta+\mathrm{Si})$ ternary eutectics and $(\mathrm{Al}+\theta)$ pseudobinary eutectics. During acoustic levitation, the $(\mathrm{Al}+\theta+\mathrm{Si})$ ternary eutectics are refined and the $(\mathrm{Al}+\theta)$ pseudobinary eutectics have morphological diversity. On the surface of the alloys, surface oscillations and acoustic streaming promote the nucleation of the three eutectic phases and expedite the cooling process. This results in the refinement of the ternary eutectic microstructure. During experiments, the reflector decreases with increasing alloy temperature, and the levitation distance always exceeds the resonant distance. Because of the acoustic radiation pressure, the melted alloy was flattened, and deformation increases with increasing sound pressure. The maximum aspect ratio achieved was 6.64 , corresponding to a sound pressure of $1.8 \times 10^{4} \mathrm{~Pa}$.
\end{abstract}

acoustic levitation, laser irradiation, rapid solidification, ternary eutectic, surface nucleation

Citation: Yan N, Geng D L, Hong Z Y, et al. Rapid solidification of acoustically levitated Al-Cu-Si eutectic alloy under laser irradiation. Chinese Sci Bull, 2011, 56: 912-918, doi: 10.1007/s11434-011-4403-5

Recently, with the rapid development of astronautics, space material sciences and technologies have become an important research subject. Because of the technical complexity and high costs of space flight, there have been limited chances to implement space experiments. Fortunately, ground simulation research provides alternative approaches for this field. Single-axis acoustic levitators can yield an acoustic radiation force with an intense acoustic field to counteract gravity. There are no special requirements regarding the electromagnetic properties of the levitated samples and can be applied to conductors and nonconductors. This includes ceramics, organic materials and molecular liquids. Therefore, it is a promising method for simulating the containerless states in space on Earth $[1,2]$. The containerless effect can be used to avoid melt contamination and is widely used in the study of melt undercooling, thermophysics and liquid droplet dynamics [3-9].

*Corresponding author (email: bbwei@nwpu.edu.cn)
Because the levitation stability is very sensitive to the physical parameters of sample and gas medium, it is difficult to perform solidification experiments of alloys with high melting temperatures. Previously, the containerless melting and solidification of low-melting point materials, such as $\mathrm{Bi}-\mathrm{Ga}$ and $\mathrm{Pb}-\mathrm{Sn}$ alloys, were performed using the single-axis acoustic levitation technique [3-5]. In this paper, we have extended the application of single-axis acoustic levitation to the containerless processing of an $\mathrm{Al}-27 \% \mathrm{Cu}-$ $5.3 \%$ Si ternary alloy, which has a higher melting point than alloys that have been investigated previously [10]. The mechanisms of rapid eutectic growth during acoustic levitation were studied. Also we considered the effects of the containerless states and ultrasonic waves.

\section{Experimental method}

The samples are prepared from pure $\mathrm{Al}$ (99.99\%), $\mathrm{Cu}$ $(99.999 \%)$ and $\mathrm{Si}(99.99 \%)$ using a laser melting furnace [11]. 
Each sample has a mass of about $0.1 \mathrm{~g}$ and its equivalent spherical radius is about $2 \mathrm{~mm}$. The main parts of the experimental setup are schematically shown in Figure 1. The single-axis acoustic levitation system was actuated using a piezoelectric transducer (independently developed), which generates ultrasonic waves with a frequency of $20 \mathrm{kHz}$. During the experiment, the sample was levitated in the first resonant state. The elastic reflector is self-adaptable, which can be used to enhance the levitation stability of the alloy [12]. To avoid the oxidation of alloy during heating and cooling, the emitter and elastic reflector were enclosed in a $\Phi 54 \times 120 \mathrm{~mm}$ quartz tube and were protected using flowing Ar gas. The heating procedure was performed using a focused Trumpf HL 1006D Nd:YAG laser (Trumpf, Germany) with a wavelength of $1064 \mathrm{~nm}$. The heating power in the experiments was $400 \mathrm{~W}$. The heating duration was $2 \mathrm{~s}$. After the laser is turned off, the levitated alloy melt cools while in the levitated state. The sample temperature was measured using a $\mathrm{PbS}$ infrared detector (independently developed), which was calibrated to true temperatures with a NiCr-NiSi thermocouple (self-manufactured).

The melting and cooling processes of the levitated alloy were recorded using a MVC1000SAC-GE30 CMOS camera (Microview, China). Figure 2 shows recorded images of the

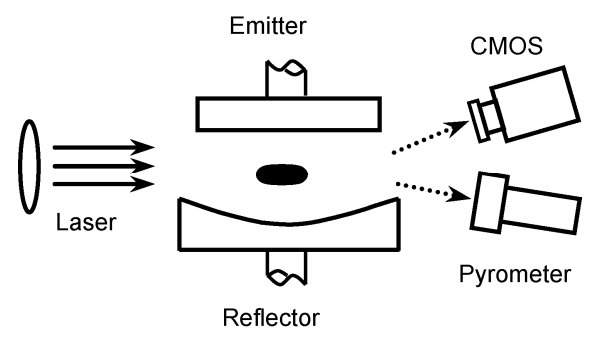

Figure 1 Schematic diagram of the acoustic levitation apparatus.

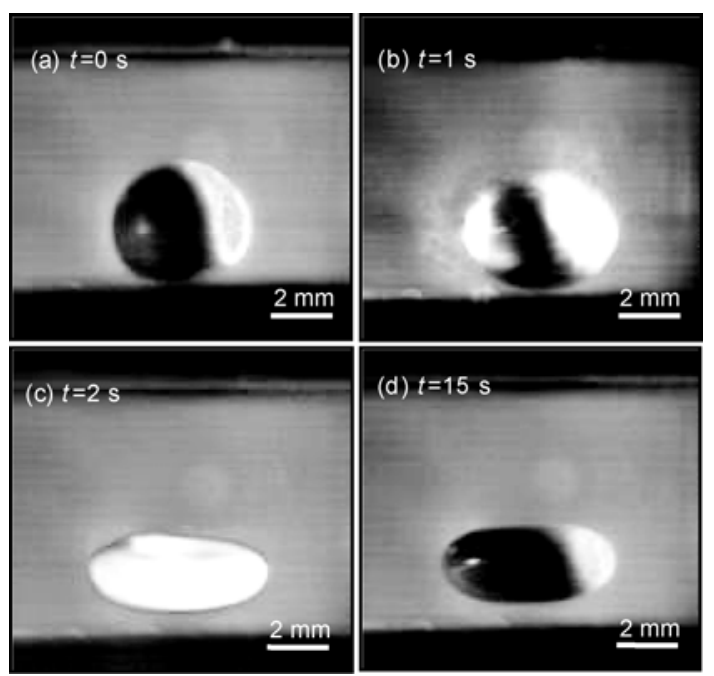

Figure 2 Containerless melting and solidification of acoustically levitated Al-27\% Cu-5.3\% Si alloy. (a) $t=0 \mathrm{~s}$, stable levitation of the solid; (b) $t=1 \mathrm{~s}$, partial melting; (c) $t=2 \mathrm{~s}$, overheated melting; (d) $t=15 \mathrm{~s}$, completely solidified. containerless processing of the $\mathrm{Al}-27 \% \mathrm{Cu}-5.3 \% \mathrm{Si}$ ternary alloy. At laser heating time $t=0 \mathrm{~s}$, the alloy is solid and is stably levitated in the gas medium. At heating time $t=1 \mathrm{~s}$, the alloy is partially melted. Once the heating time reaches $2 \mathrm{~s}$, the alloy is completely melted, and the central part of its top and bottom surfaces changes from a convex shape to a concave shape. This can be seen in Figure 2(c). After the laser is turned off, the temperature of the alloy and gas medium decrease continuously and the pancake-like deformation is still present after the alloy has completely solidified ( $t=15 \mathrm{~s})$. In Figure 2(d), it can be seen that the equatorial radius, $a$, of the deformed alloy is $2.85 \mathrm{~mm}$, and the polar radius $b$ is $1.1 \mathrm{~mm}$, which correspond to an aspect ratio $(a / b)$ of 2.59 . From the experimental results of the containerless processing using this improved acoustic levitator, we concluded that elastic reflector can offer a self-adaptive adjustment to the temperature. It is a promising method for enhancing the levitation stability during solidification.

After the experiments, the solidified samples were dissected, polished and etched. The thermodynamic properties, were analyzed using a Netzsch DSC 404C differential calorimeter (Netzsch, Germany), the phase constitutions with a Rigaku D/max $2500 \mathrm{~V}$ X-ray diffractometer (Rigaku, Japan), the microstructures with a Zeiss Axiovert 200 MAT optical microscope (Carl Zeiss, Germany) and the solute distributions with an Inca 300 energy dispersive spectroscope (Oxford Instruments, UK).

\section{Results and discussion}

\subsection{Cooling curve and levitation distance}

Figure 3(a) shows a cooling curve of the Al-27\% Cu-5.3\% $\mathrm{Si}$ ternary alloy. In this experiment, a maximum undercooling of $195 \mathrm{~K}\left(0.24 T_{\mathrm{L}}\right)$ was achieved, and the average cooling rate was $76 \mathrm{~K} / \mathrm{s}$.

The temperature of the gas medium varies with the melting and solidification of the alloy. This temperature variation results in changes in the medium density and acoustic wavelength. Therefore, instabilities are introduced into the acoustic levitation [13]. Here, we define the levitation distance as $H$, which denotes the distance between the emitter and the center of reflector. By analyzing the recorded images in Figure 2 with a digital image processing method, the levitation distance $H$ was determined for different temperatures. According to Figure 3(b), the levitation distance $H$ increases with the rising temperature. The calculation of the resonant distance, $H_{\mathrm{r}}$, is based on the work of Xie et al. [14]. $H_{\mathrm{r}}$ is calculated and plotted with dashed line in Figure 3(b). The resonant distance, $H_{\mathrm{r}}$, also increases with increasing temperature, and is always smaller than the levitation distance $H$. This indicates that the levitation distance should be larger than the resonant distance for the stable levitation of 

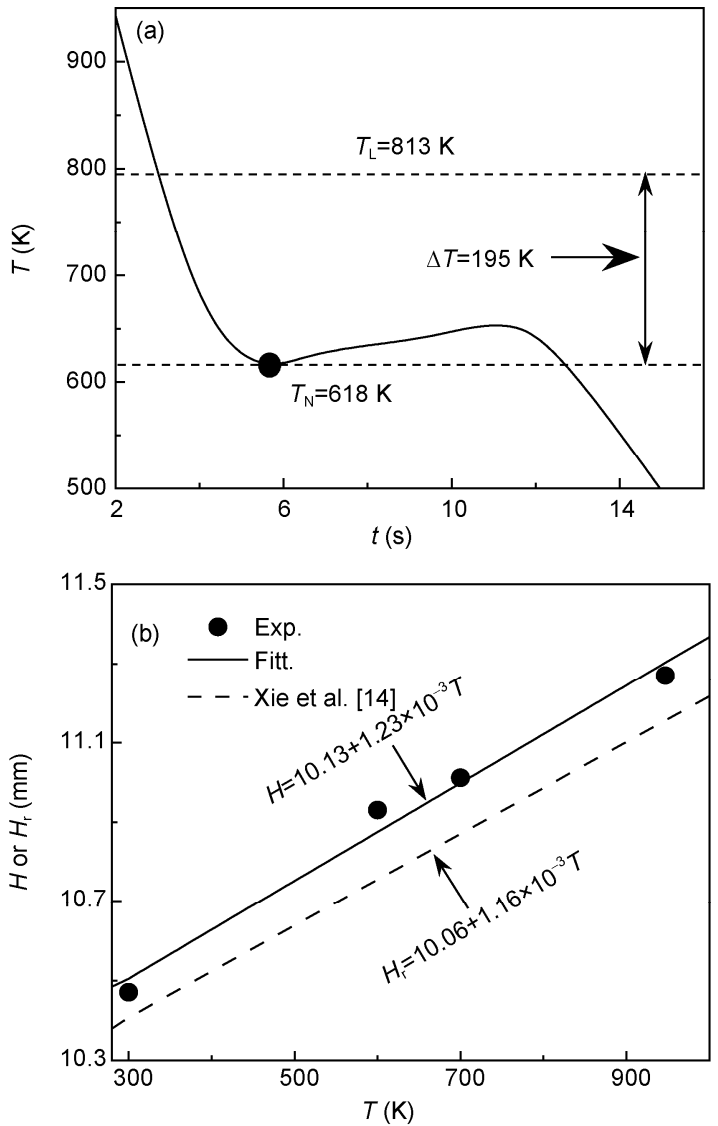

Figure 3 Cooling curve together with levitation and resonant distances. (a) Cooling curve; (b) levitation and resonant distances.

the alloy during containerless processing [15].

\subsection{Droplet deformation mechanism}

The physical origin of the droplet deformation is the acoustic radiation pressure, $P_{\mathrm{r}}$, which is a nonlinear effect of high intensity sound or ultrasound. King's theory [16] shows that a substantial time-averaged acoustic radiation pressure results from the second-order terms of the sound pressure $p$ and the medium particle velocity $v$, which can be expressed as

$$
P_{\mathrm{r}}=\frac{1}{2} \frac{\rho_{0}}{c_{0}^{2}}\left\langle p^{2}\right\rangle-\frac{1}{2} \rho_{0}\left\langle v^{2}\right\rangle,
$$

where $\rho_{0}$ and $c_{0}$ are the medium density and speed of sound, respectively. The angular brackets in eq. (1) denote a time average over one period of acoustic oscillation. The integral of the acoustic radiation pressure over the entire surface of the alloy contributes the acoustic radiation force for levitation. Based on the spheroidal wave functions, the acoustic radiation pressure is given by the following equations [17]:

$$
P_{\mathrm{r}}=P_{\mathrm{s}}+P_{1},
$$

where

$$
\begin{aligned}
P_{\mathrm{s}}= & \frac{p_{\mathrm{A}}{ }^{2}}{4 \rho_{0} c_{0}^{2}}\left[\sin ^{2} k x-\frac{4}{\pi^{2}} \cos ^{2} k x \frac{(r / a)^{2}}{\left(1-r^{2} / a^{2}\right)}\right. \\
& \left.+\frac{4}{\pi^{2}}\left(k R_{\mathrm{s}}\right)^{2} \cos ^{2} k x\left(1-\frac{r^{2}}{a^{2}}\right)\right]
\end{aligned}
$$

is symmetric with respect to the top and bottom of the deformed droplet, and is responsible for determining its shape.

$$
P_{1}= \pm\left(k R_{\mathrm{s}} p_{\mathrm{A}}{ }^{2} / 2 \pi \rho_{0} c_{0}^{2}\right)\left(1-\frac{r^{2}}{a^{2}}\right)^{\frac{1}{2}} \sin 2 k x
$$

is antisymmetric, and is responsible for the droplet levitation. Here, $p_{\mathrm{A}}$ is the sound pressure amplitude, $k$ is the wave number, $x$ is the vertical coordinate of the levitated droplet, $R_{\mathrm{s}}$ is the equivalent spherical radius, and $r$ is the radial position on the droplet surface.

Figure 4(a) shows the distribution of the acoustic radiation pressure on the alloy sample surface. There are both positive and negative pressures on both the top and the bottom. In the center of the alloy sample, the acoustic radiation pressure at the top and bottom are $4.5 \times 10^{-4}$ and $30 \mathrm{~Pa}$, respectively. On the sides of the alloy sample, the acoustic radiation pressure of sample top and bottom is the same and is $-1.7 \times 10^{2} \mathrm{~Pa}$. Here, the positive pressure on the central regions of sample top and bottom compresses the objection. However, the negative pressure on the peripheral regions has a suction effect. Therefore, the acoustic radiation pressure on the alloy surface has a tendency to flatten the alloy sample. The molten alloy deforms into different shapes, such as ellipsoids or pancake-like structures, immediately after the alloy is transformed from solid to liquid.

The equilibrium shape of the levitated droplet is determined by the balance between the acoustic radiation pressure and the surface tension of the alloy. Marston [18] theoretically evaluated the effects of the acoustic radiation pressure on a compressible sphere using a linear and inviscid theory. It gives the equilibrium shape of a levitated droplet as

$$
r_{\mathrm{s}}(\theta)=R_{\mathrm{s}}\left[1-\frac{3 R_{\mathrm{s}} p_{\mathrm{A}}^{2}}{64 \gamma \rho_{0} c_{0}^{2}}\left(1+\frac{7}{5}\left(k R_{\mathrm{s}}\right)^{2}\right)\left(3 \cos ^{2} \theta-1\right)\right],
$$

where $r_{\mathrm{s}}$ is the distance from the point of the droplet surface to the droplet origin, $\theta$ is the polar angle with respect to the vertical axis and $\gamma$ is the surface tension of the alloy. Here, the aspect ratio, $a / b=r_{\mathrm{s}}(\pi / 2) / r_{\mathrm{s}}(0)$, is studied to evaluate the deformation of the levitated droplets. According to eq. (5), the deformation of the droplet primarily depends on the sound pressure amplitude.

When a droplet is acoustically levitated, the sound pressure amplitude of the acoustic field, $p_{\mathrm{A}}$, must satisfy the condition $p_{\mathrm{m}} \leqslant p_{\mathrm{A}} \leqslant p_{\mathrm{M}}$. Here, $p_{\mathrm{m}}$ is the minimum pressure 

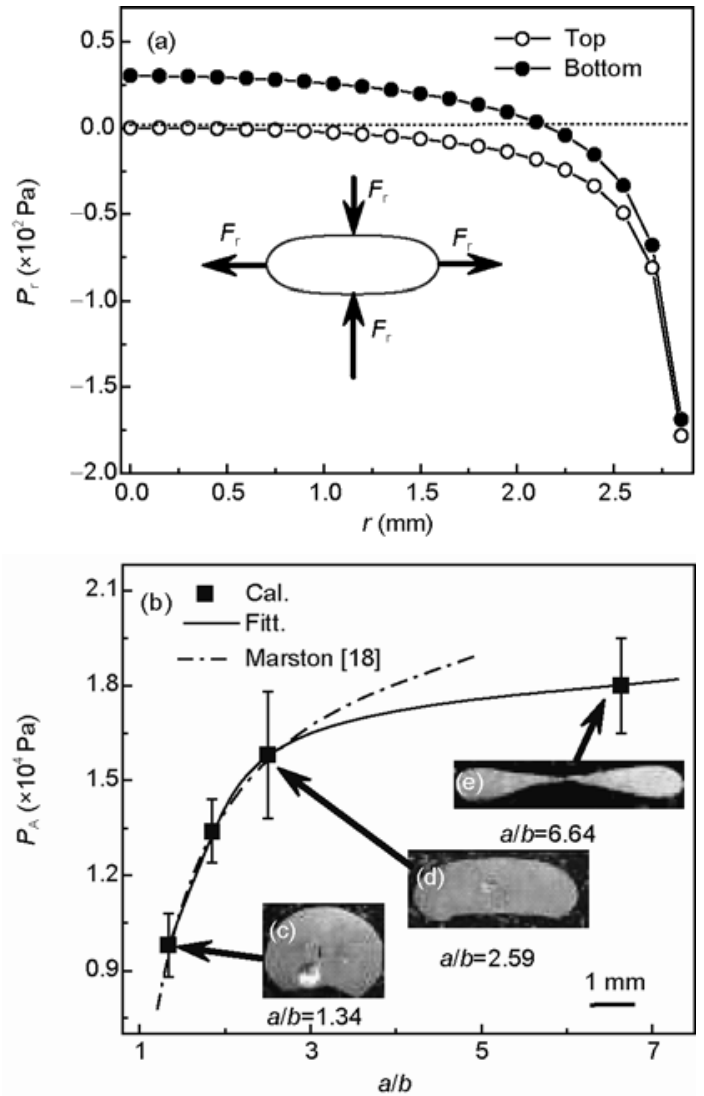

Figure 4 Acoustic radiation pressure and sound pressure. (a) Acoustic radiation pressure; (b) sound pressure; (c), (d) and (e) the macrostructure of the deformed samples.

needed to levitate the droplet, and $p_{\mathrm{M}}$ is the maximum pressure needed to ensure the droplet stability [14,17]. For Al-27\%Cu-5.3\% Si ternary alloy, $p_{\mathrm{m}}$ and $p_{\mathrm{M}}$ are $0.8 \times 10^{4}$ and $1.8 \times 10^{4} \mathrm{~Pa}$, respectively. From the deformation of the alloy sample, the sound pressure amplitude was derived using eq. (5). This is shown in Figure 4(b). When the aspect ratio $a / b$ of the alloy is equal to 1.34, which can be seen in Figure 4(c), the sound pressure amplitude is about $1.0 \times 10^{4} \mathrm{~Pa}$. When the sound pressure is increased, the droplet becomes flattener. It took on a pancake-like shape, which is shown in Figure 4(d). At a higher sound pressures, the central parts of its top and bottom surface change from a convex shape to a concave shape, which can be seen in Figure 4(e). At the aspect ratio of 6.64 , the corresponding sound pressure amplitude nearly reaches the maximum threshold $p_{\mathrm{M}}$. The aspect ratio of alloy sample increases with the rising sound pressure.

\subsection{Microstructural characteristics}

A thermal analysis of the Al-27\% Cu-5.3\% Si ternary alloy was performed using differential scanning calorimetry (DSC). A sample with a mass of $21.59 \mathrm{mg}$ was heated and cooled at a rate of $10 \mathrm{~K} / \mathrm{min}$ in an Ar gas environment. The measured DSC curve is shown in Figure 5(a). Its total
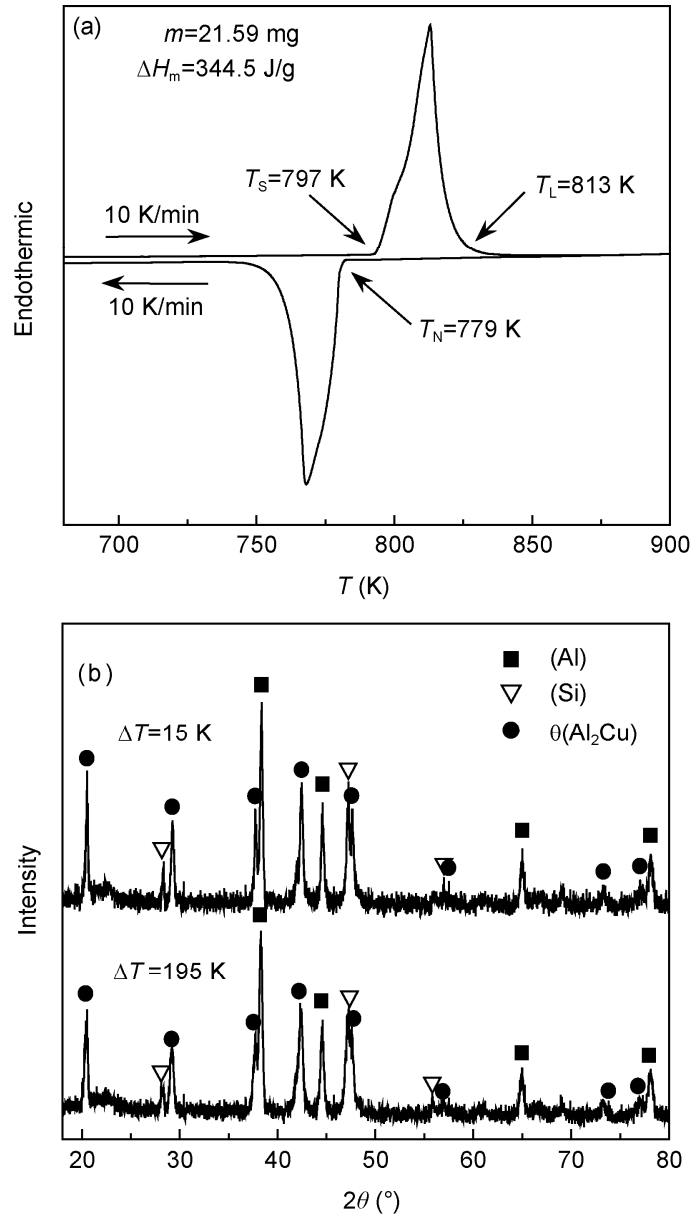

Figure 5 Thermal analysis and X-ray diffraction of $\mathrm{Al}-27 \% \mathrm{Cu}-5.3 \% \mathrm{Si}$ ternary alloy. (a) DSC thermogram; (b) XRD patterns.

melting enthalpy was determined as $344.5 \mathrm{~J} / \mathrm{g}$, and its liquidus and solidus temperatures were 813 and $797 \mathrm{~K}$, respectively.

To investigate the solidification characteristics of the material under acoustic levitation, the master alloy was placed in an alumina crucible to create a conventionally solidified sample for comparison. X-ray diffraction (XRD) analyses were performed on the samples solidified using the two different methods. The XRD patterns are shown in Figure 5(b). No metastable phases other than (Al) solid solution, $\theta\left(\mathrm{Al}_{2} \mathrm{Cu}\right)$ intermetallic compound and ( $\mathrm{Si}$ ) semiconductor phase were found in either of the two samples.

Figures 6 and 7 show the solidification morphologies of the Al-27\% Cu-5.3\% Si ternary alloy solidified using the two methods. Here, the white phase is the (Al) phase, the grey phase is the $\theta\left(\mathrm{Al}_{2} \mathrm{Cu}\right)$ phase, and the black phase is the $(\mathrm{Si})$ phase. When conventional solidification condition was used, the alloy undercooling was only $15 \mathrm{~K}$. The solidified microstructure is composed of $(\mathrm{Al}+\theta+\mathrm{Si})$ ternary eutectics and $(\mathrm{Al}+\theta)$ pseudobinary eutectics. This can be seen in Figures 6(a) and 7(a). In the $(\mathrm{Al}+\theta+\mathrm{Si})$ ternary eutectics, the $(\mathrm{Al})$ and $\theta$ phases grow cooperatively, while 


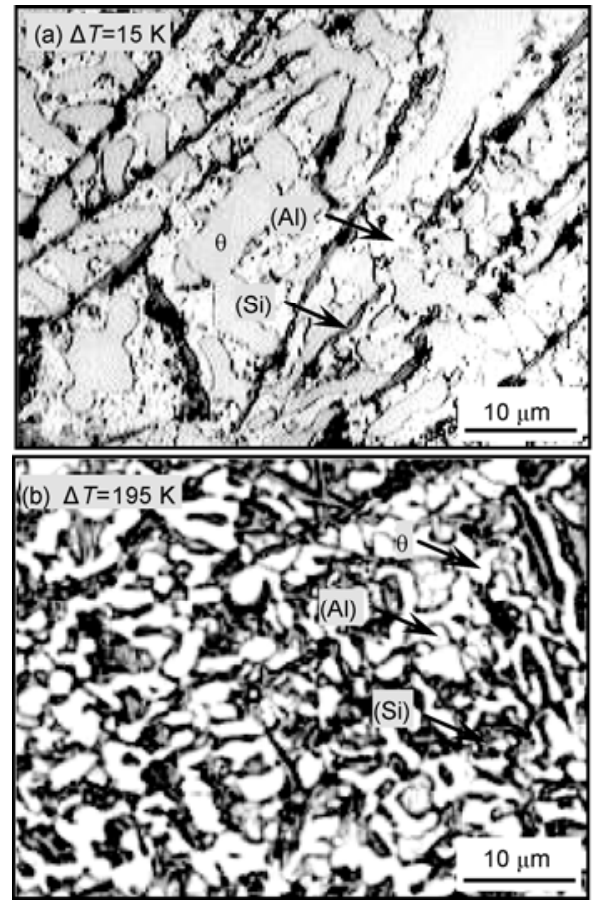

Figure 6 Growth morphology of the $(\mathrm{Al}+\theta+\mathrm{Si})$ ternary eutectics. (a) Solidified using the conventional method; (b) solidified during acoustic levitation.

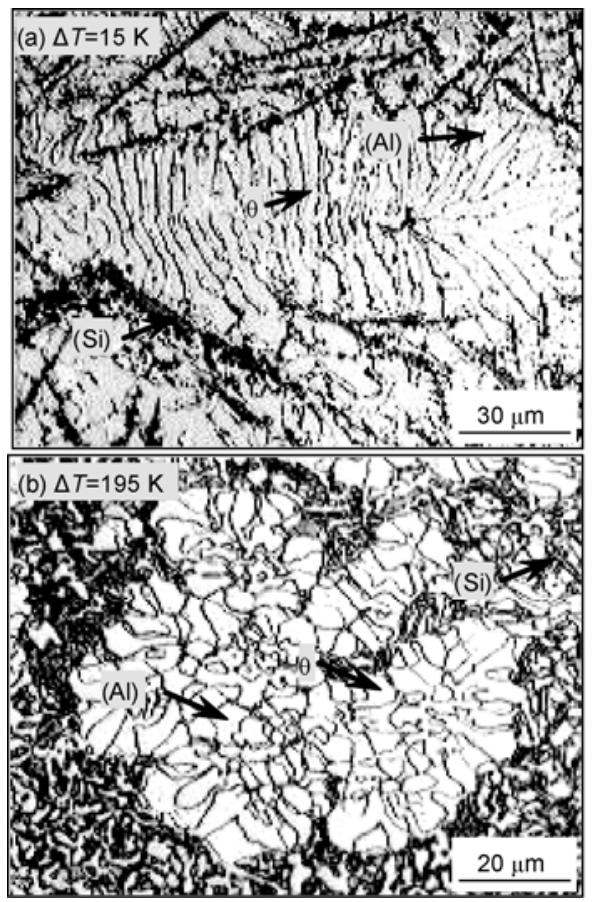

Figure 7 Morphologies of the $(\mathrm{Al}+\theta)$ pseudobinary eutectics. (a) Solidified using the conventional method; (b) solidified during acoustic levitation.

the faceted $(\mathrm{Si})$ phase grows like a needle with a preferred growth orientation. The average length of the ( $\mathrm{Si}$ ) phase was $56 \mu \mathrm{m}[10]$.

The (Al) and $\theta$ phases display strong adherence and grow in the form of alternating lamellae like fish bones. The corresponding average pseudobinary eutectic spacing is about $8.6 \mu \mathrm{m}$. When the alloy was solidified during acoustic levitation, the maximum alloy undercooling was $\Delta T=195 \mathrm{~K}$. The $(\mathrm{Al}+\theta+\mathrm{Si})$ ternary eutectics become finer and the cooperative growth between the $(\mathrm{Al})$ and $\theta$ phases weakens. In addition, the average length of the ( $\mathrm{Si}$ ) phases was reduced to $12 \mu \mathrm{m}$, and the growth of the ( $\mathrm{Si}$ ) phase loses its local preferred orientation. This can be seen in Figure 6(b). Furthermore, the morphology of the $(\mathrm{Al}+\theta)$ pseudobinary eutectics becomes diverse, forming flowerlike or cluster structures. Also, the average pseudobinary eutectic spacing decreases to $5.8 \mu \mathrm{m}$. This can be seen in Figure 7(b). Energy dispersive spectrometry was applied to determine the solute content in the $(\mathrm{Al})$ and $\theta$ phases formed using the two cooling methods. The maximum solubility of $\mathrm{Cu}$ and $\mathrm{Si}$ in the $(\mathrm{Al})$ phase is $4.4 \mathrm{wt} \%$ and $0.72 \mathrm{wt} \%$ using the conventional solidification method. However, the maximum solubility in the $(\mathrm{Al})$ phase is $8.1 \mathrm{wt} \% \mathrm{Cu}$ and $1.4 \mathrm{wt} \% \mathrm{Si}$ when the sample is acoustically levitated, which is double that of conventional solidification method. However, the maximum solubility of $\mathrm{Si}$ in the $\theta\left(\mathrm{Al}_{2} \mathrm{Cu}\right)$ phase is $0.79 \mathrm{wt} \%$ and $0.98 \mathrm{wt} \%$ when the conventional and acoustic levitation methods are used, respectively. The variation in the maximum solubility of the $\theta\left(\mathrm{Al}_{2} \mathrm{Cu}\right)$ intermetallic compound is relatively small. This demonstrates that containerless state can enhance the undercooling of the alloy, refine the solidification microstructure, and extend the solubility of the phases.

Using the acoustic boundary condition that the sound pressure is continuous at the interfaces of the two media, we estimated the sound pressure inside the $\mathrm{Al}-27 \% \mathrm{Cu}-5.3 \% \mathrm{Si}$ alloy to be the same order of magnitude as that on the surface, i.e. $\sim 1 \times 10^{4} \mathrm{~Pa}$. Also, the acoustic characteristic impedance of the $\mathrm{Al}-27 \% \mathrm{Cu}-5.3 \% \mathrm{Si}$ alloy is about $1.9 \times 10^{7} \mathrm{Ns} / \mathrm{m}^{3}$, which is much larger than that of the gas medium $\left(4.4 \times 10^{2} \mathrm{Ns} / \mathrm{m}^{3}\right)$. The sound energy inside the alloy was estimated to be $1 / 22000$ that in the gas medium. This indicates that $99.9955 \%$ of the sound energy was reflected away from the alloy surface into the gas medium. Therefore, the variation in sound pressure and the deformation of the alloy have little influence on the internal solidification process during acoustic levitation. However, the sound pressure can affect the surface area of the alloy because of accompanying effects, such as surface oscillation and acoustic cavitation [4,5].

Figure 8 shows the eutectic growth morphology on the surface of the levitated sample. The $(\mathrm{Al}+\theta+\mathrm{Si})$ ternary eutectic is finer and denser than that in the inside of the sample. Both the (Al) and $\theta$ phases have a globular shape without preferred growth orientations. However, the (Si) phase grows like a worm. Figures 8(b) and (c) are the enlargements of areas A and B in Figure 8(a), respectively. The average pseudobinary eutectic spacing was about 1 and 4 $\mu \mathrm{m}$ in areas $\mathrm{A}$ and $\mathrm{B}$, respectively. It is evident that the 

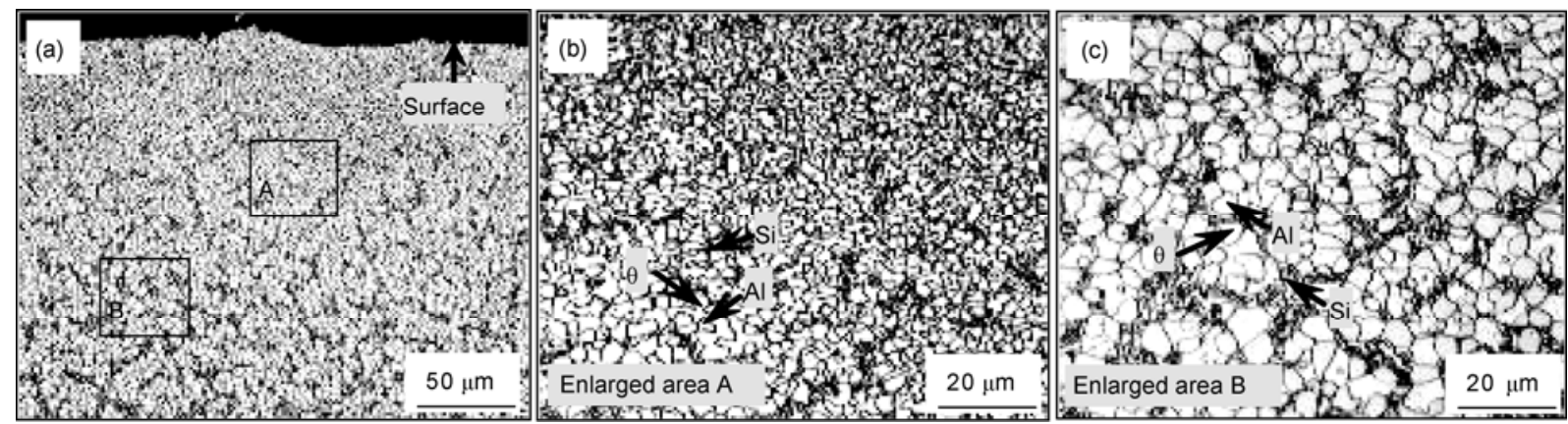

Figure 8 Surface morphology characteristics of the acoustically levitated Al-27\%Cu-5.3\%Si alloy with an undercooling of $\Delta T=195 \mathrm{~K}$. (a) Macrostructure; (b) enlargement of area A; and (c) enlargement of area B.

average grain size of the pseudobinary eutectic increases and the number of nuclei per volume decreases gradually as one moves from area A to area B. Using the above-mentioned solidification characteristics, we conclude that the sound pressure has a drastic effect on the alloy surface, and that the influence decreases gradually as the distance from the alloy surface increases. The solidification mechanism of the surface of the $\mathrm{Al}-27 \% \mathrm{Cu}-5.3 \% \mathrm{Si}$ alloy under acoustic levitation can be explained as follows. First, the sound pressure excites oscillations and energy fluctuations. These introduce the additional energy for nucleation and increase the formation probability of nuclei on the alloy surface [4]. Second, the intense sound from the acoustic levitation causes many nonlinear effects. One of them is acoustic streaming, which increases the heat transfer coefficient by as much as a factor of four $[19,20]$. This large heat transfer coefficient accelerates the process of thermal exchange and rapidly reduces the temperature of the alloy surface. This suggests that the undercooling and cooling rate were enhanced, and the resultant nucleation and growth were improved. According to the analysis above, we conclude that the sound field has strong effects on the nucleation and growth of the three eutectic phases on the surface of the alloy sample.

\section{Conclusions}

The containerless melting and solidification of $\mathrm{Al}-27 \% \mathrm{Cu}-$ $5.3 \% \mathrm{Si}$ ternary eutectic alloy, which has a melting point of $813 \mathrm{~K}$, was successfully accomplished using single-axis acoustic levitation with an elastic reflector and YAG laser beam heating system. The elastic reflector offers a selfadaptive adjustment to the varying temperature. It is a promising method to enhance levitation stability during containerless processing. The maximum undercooling was $195 \mathrm{~K}\left(0.24 T_{\mathrm{L}}\right)$ and the cooling rate was about $76 \mathrm{~K} / \mathrm{s}$. The microstructure is composed of $(\mathrm{Al})$ solid solution, $\theta\left(\mathrm{Al}_{2} \mathrm{Cu}\right)$ intermetallic compound and ( $\mathrm{Si}$ ) semiconductor after cooling during acoustic levitation or conventional condition. The (Al) and $\theta$ phases grow cooperatively to form the $\left(\mathrm{Al}^{+}+\theta\right)$ pseudobinary eutectic. It exhibits morphological diversity, and the average spacing of the $(\mathrm{Al})$ and $\theta$ phases decreases to $5.8 \mu \mathrm{m}$. In the $(\mathrm{Al}+\theta+\mathrm{Si})$ ternary eutectic, the average length of the ( $\mathrm{Si}$ ) phase reduces to $12 \mu \mathrm{m}$. On the surface of the alloy sample, the $(\mathrm{Al}+\theta+\mathrm{Si})$ ternary eutectics are refined. This indicates that surface oscillations and acoustic streaming have important roles in promoting the nucleation and growth of the three eutectic phases on the surface. During the experiment, the levitation distance and the resonant distance increased with increasing alloy temperature. The levitation distance must be larger than the resonant distance for the stable levitation of the alloy sample during containerless processing. With rising sound pressure, the acoustic radiation pressure and the deformation of the sample also increase. The maximum aspect ratio of 6.64 corresponds to a sound pressure of $1.8 \times 10^{4} \mathrm{~Pa}$.

The authors express their many thanks to Prof. Xie $W J, D r$. Ruan $Y, D r$. Dai F P and Dr. Wang W L for their help. This work was supported by the National Natural Science Foundation of China (50971105).

1 Catherall A T, Eaves L, King P J, et al. Magnetic levitation: Floating gold in cryogenic oxygen. Nature, 2003, 422: 579

2 Tuckermann R, Neidhart B, Lierke E G, et al. Trapping of heavy gases in stationary ultrasonic fields. Chem Phys Lett, 2002, 363: 349-354

3 Hong Z Y, Lü Y J, Xie W J, et al. The liquid phase separation of $\mathrm{Bi}-\mathrm{Ga}$ hypermonotectic alloy under acoustic levitation condition. Chinese Sci Bull, 2007, 52: 1446-1450

4 Lü Y J, Xie W J, Wei B. Observation of ice nucleation in acoustically levitated water drops. Appl Phys Lett, 2005, 87: 184107

5 Ohsaka K, Trinh E H, Glicksman M E. Undercooling of acoustically levitated molten drops. J Cryst Growth, 1990, 106: 191-196

6 Tian Y, Holt R G, Apfel R E. A new method for measuring liquid surface tension with acoustic levitation. Rev Sci Instrum, 1995, 66: 3349-3354

7 Bauerecker S, Neidhart B. Formation and growth of ice particles in stationary ultrasonic fields. J Chem Phys, 1998, 109: 3709-3712

8 Santesson S, Nilsson S. Airborne chemistry: Acoustic levitation in chemical analysis. Anal Bioanal Chem, 2004, 378: 1704-1709

9 Wulsten E, Lee G. Surface temperature of acoustically levitated water microdroplets measured using infrared thermography. Chem Eng Sci, 2008, 63: 5420-5424

10 Ruan Y, Wei B. Rapid solidification of undercooled Al-Cu-Si eutectic alloys. Chinese Sci Bull, 2009, 54: 53-58 
11 Richard Weber J K, Felten J J, Nordine Paul C. Laser hearth melt processing of ceramic materials. Rev Sci Instrum, 1996, 67: $522-524$

12 Hong Z Y, Xie W J, Wei B. Vibration characteristics of acoustically levitated object with rigid and elastic reflectors. Chin Phys Lett, 2010, 27: 014301

13 Leung E W, Wang T G. Force on a heated sphere in a horizontal plane acoustic standing wave field. J Acoust Soc Am, 1985, 77: 1686-1691

14 Xie W J, Wei B. Temperature dependence of single-axis acoustic levitation. J Appl Phys, 2003, 93: 3016-3021

15 Xie W J, Wei B. Dynamics of acoustically levitated disk samples.
Phys Rev E, 2004, 70: 046611

16 King L V. On the acoustic radiation pressure on spheres. Proc Roy Soc, 1934, A147: 212-240

17 Lee C P, Anilkumar A V, Wang T G. Static shape and instability of an acoustically levitated liquid drop. Phys Fluids A, 1991, 3: 2497-2516

18 Marston P L. Shape oscillation and static deformation of drops and bubbles driven by modulated radiation stresses-Theory. J Acoust Soc Am, 1980, 67: 15-26

19 Zhao H, Sadhal S S, Trinh E H. Internal circulation in a drop in an acoustic field. J Acoust Soc Am, 1999, 106: 3289-3295

20 Fand R M. Mechanism of interaction between vibrations and heat transfer. J Acoust Soc Am, 1962, 34: 1887-1894

Open Access This article is distributed under the terms of the Creative Commons Attribution License which permits any use, distribution, and reproduction in any medium, provided the original author(s) and source are credited. 